\title{
Membrana neovascular sub-retineana justapapilar em paciente com papiledema e hipertensão intracraniana idiopática
}

\section{Juxtapapillary subretinal neovascular membrane in a patient with papilledema and idiopathic intracranial hyperthension}

Mário Luiz Ribeiro Monteiro', Martha de Queiroz Monteiro Jales²,Sérgio Luis Gianotti Pimentel ${ }^{3}$

\begin{abstract}
Resumo
Este trabalho descreve o caso de uma paciente de 41 anos, com diagnóstico da síndrome da hipertensão intracraniana idiopática (síndrome do pseudotumor cerebral) que desenvolveu perda visual em um dos olhos decorrente de membrana neovascular subretiniana envolvendo a região macular. Apesar do tratamento clínico adequado e da realização de fenestração da bainha do nervo óptico com boa regressão do edema de papila não houve resolução da membrana neovascular sub-retiniana mesmo após três injeções intravítreas seqüenciais de bevacizumab. Chamamos a atenção para esta complicação incomum do papiledema, revisamos a literatura a este respeito e discutimos as possíveis causas, bem como a evolução mais comum e as modalidades de tratamento disponíveis para tratamento da membrana neovascular sub-retiniana em pacientes com a síndrome do pseudotumor cerebral.
\end{abstract}

Descritores: Pseudotumor cerebral/complicações; Neovascularização retiniana/ fisiopatologia; Neovascularização retiniana/terapia; Papiledema/complicações; Acuidade visual; Relatos de casos

\footnotetext{
'Livre-docente, Professor associado da Faculdade de Medicina da Universidade de São Paulo - USP - São Paulo (SP), Brasil; ${ }^{2}$ Residente de terceiro ano da Divisão de Clínica Oftalmológica da Faculdade de Medicina da Universidade de São Paulo - USP - São Paulo (SP), Brasil;

${ }^{3}$ Médico Colaborador da Divisão de Clínica Oftalmológica da Faculdade de Medicina da Universidade de São Paulo - USP - São Paulo (SP), Brasil.
}

Recebido para publicação em: 13/1/2009 - Aceito para publicação em 1/2/2009 


\section{INTRODUÇÃO}

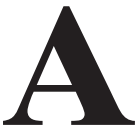
síndrome da hipertensão intracraniana idiopática (HII) é uma afecção caracterizada por sinais e sintomas de aumento da pressão intracraniana, ausência de lesões expansivas e de ventriculomegalia aos exames de neuroimagem, e constituição do líquor normal em um paciente sem alterações no nível de consciência ${ }^{(1)}$. Embora seja uma condição relativamente benigna do ponto de vista neurológico, a HII pode levar a inúmeras alterações oftalmológicas acarretando perda visual em uma porcentagem muito alta de indivíduos. O mecanismo mais comum da perda visual é a atrofia progressiva das fibras nervosas da papila associada à constrição difusa do campo visual ${ }^{(2,3)}$. Muito menos comumente a perda visual pode ocorrer por complicações hemorrágicas retinianas $^{(4 \cdot)}$.

O objetivo deste trabalho é relatar uma paciente com HII que apresentou com perda visual em ambos os olhos, decorrente de membrana neovascular subretiniana justapapilar, revisar outros casos semelhantes na literatura e discutir o provável mecanismo de desenvolvimento e tratamento desta complicação incomum do papiledema.

\section{Relato do caso}

Paciente de 41 anos, feminina, parda, procedente da zona rural do estado de Minas Gerais, foi encaminhado ao serviço de Neuroftalmologia do Hospital das Clínicas da Faculdade de Medicina da Universidade de São Paulo com diagnóstico de HII, por apresentar perda visual. Relatava história de cefaléia frontal e retroorbitária de forte intensidade, contínua e pior à noite; associada a embaçamento visual do olho direito (OD) há 6 meses. Procurou médico que solicitou exames de tomografia computadorizada e imagem por ressonância magnética de crânio que foram normais e exame de líquor que evidenciou hipertensão intracraniana, sendo o restante do exame normal. Havia sido feito então o diagnóstico de HII e iniciado o tratamento com acetazolamida ( $250 \mathrm{mg}$ por via oral de $6 / 6 \mathrm{~h}$ ) e topiramato (50mg via oral 2 vezes ao dia) sem melhora dos sintomas. Nos antecedentes referia obesidade, ser portadora de mioma, ter realizado mamoplastia 13 anos antes e ter feito colecistectomia há 1 mês. Negava tabagismo, etilismo ou uso de medicações.

Ao exame oftalmológico, a acuidade visual (AV) corrigida era 0,15 no OD (com - $0,50 \mathrm{DC}$ a $30^{\circ}$ ) e 1,0 no olho esquerdo $(\mathrm{OE})\left(\mathrm{com}-0,50 \mathrm{DC}\right.$ a $\left.110^{\circ}\right)$. As pupilas eram isocóricas e havia um defeito pupilar aferente re-
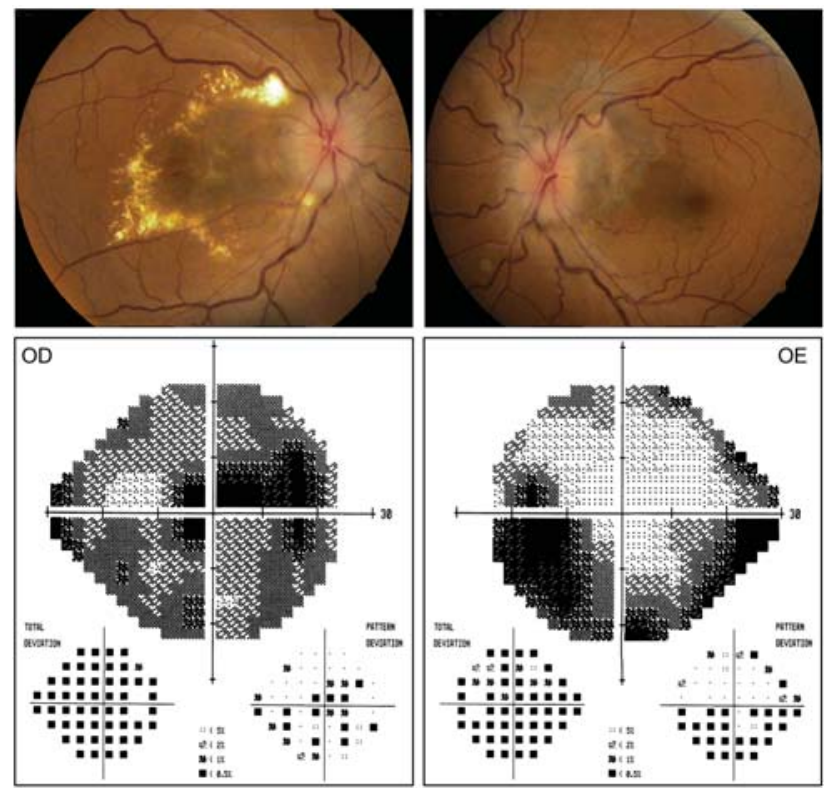

Figura 1: Retinografia demonstrando papiledema bilateral com exsudatos duros na região macular do olho direito (OD) e alteração pigmentar peripapilar no olho esquerdo (OE). Abaixo, campo visual computadorizado (Humphrey, 24-2 SITA Standard test) evidenciando constrição difusa e escotoma cecocentral no OD e constrição periférica mais evidente inferiormente além de aumento da mancha cega no OE

lativo discreto à direita. $\mathrm{O}$ exame da motilidade ocular extrínseca e a biomicroscopia do segmento anterior foram normais e a pressão intra-ocular foi $12 \mathrm{mmHg}$ em cada olho. Ao fundo de olho (FO), observou-se edema de disco óptico associado a tortuosidade vascular em cada olho, edema e exsudatos maculares no OD e discreta alteração pigmentar peripapilar temporal superior no OE (Figura 1). O campo visual (Humphrey, 24-2 SITA Standard test) mostrou no OD depressão importante do campo associado à escotoma cecocentral e no $\mathrm{OE}$ constrição periférica mais acentuada no setor inferior associado a aumento da mancha cega (Figura 1). Foram realizadas angiofluoresceinografia e a tomografia de coerência óptica que demonstraram extravasamento de contraste da papila em ambos os olhos e documentaram a existência de membrana neovascular sub-retiniana clássica no $\mathrm{OD}$ e alterações pigmentares peripapilares no OE (Figura 2). A angiografia por indocianina verde confirmou os achados demonstrando um ponto de vazamento ("hot spot") na região macular próximo à fóvea.

Após internação hospitalar a paciente foi submetida à punção liquórica lombar que revelou pressão inicial de $40 \mathrm{cmH} \mathrm{O}$ (normal até 20) e final de $22 \mathrm{cmH}_{2} \mathrm{O}$, com aspecto límpido e incolor. A análise do líquido cefalorraquidiano mostrou contagem global de células de 1 por $\mathrm{mm}^{3}$ ( $91 \%$ linfomononucleares) e 4 hemácias 

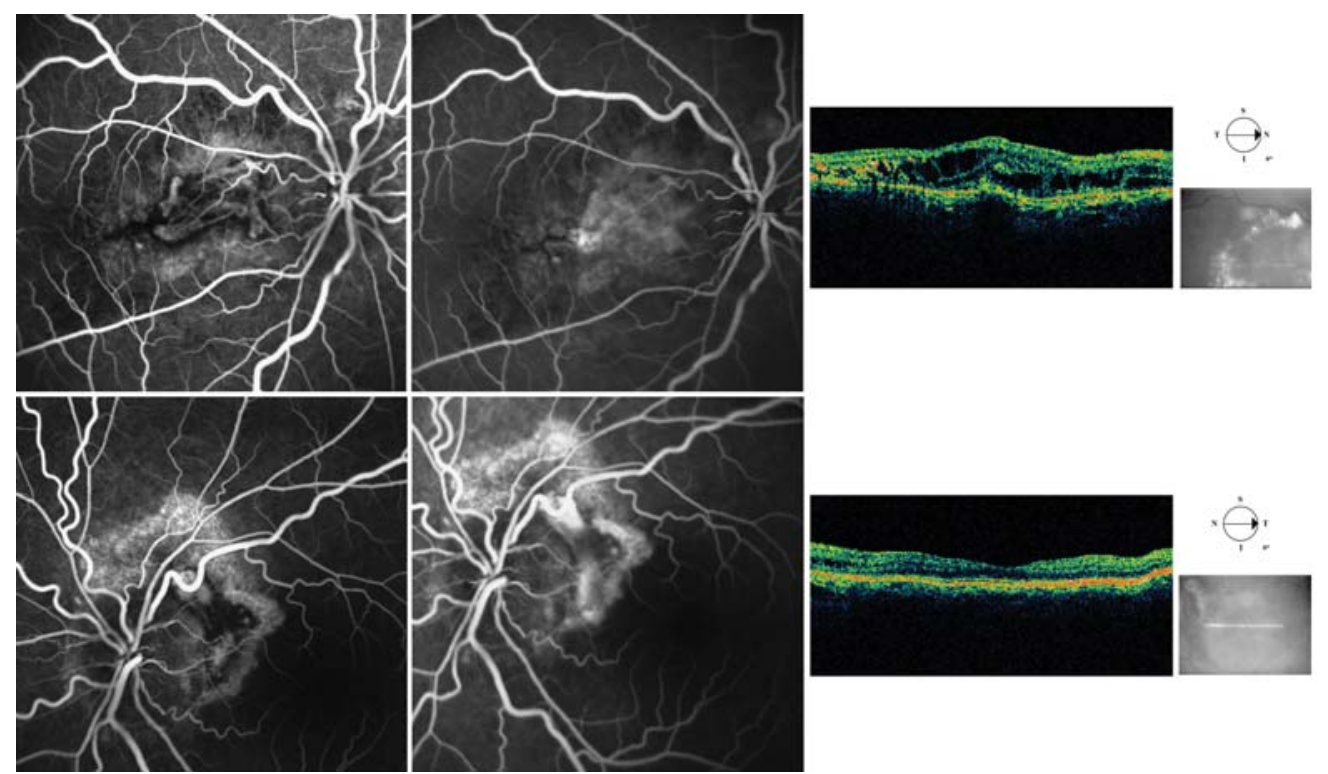

Figura 2: Angiofluoresceinografia de fase precoce (à esquerda), de fase tardia (centro) e tomografia de coerência óptica (à direita) evidenciando membrana neovascular subretiniana no olho direito (acima) e cicatriz peripapilar temporal superior sem neovascularização no olho esquerdo (abaixo). Observe o aumento da espessura na região macular com perda da depressão foveal e espaços císticos intrarretinianos à tomografia de coerência óptica no olho direito (acima e à direita)

$\mathrm{mm}^{3}$.A citologia oncótica, dosagem de cloretos, glicose, uréia e proteínas $(23 \mathrm{mg} / \mathrm{dl})$ apresentaram valores normais. A paciente foi submetida à imagem por IRM de crânio e órbitas e angiorressonância magnética venosa de crânio que foram normais. Exames laboratoriais incluindo hemograma, coagulograma, dosagem sérica de uréia e creatinina, velocidade de hemossedimentação e as sorologias para HIV, sífilis, vírus HTLV, toxoplasmose e citomegalovirus nada revelaram.

Com a confirmação do diagnóstico de HII e constatada a presença de membrana neovascular subretiniana no OD e exsudação retiniana importante foi aumentada dose da acetazolamida para 1,5 g/d e 15 dias depois realizada injeção intravítrea de bevacizumab $1,25 \mathrm{mg}(0,05 \mathrm{ml})$ no OD. Como não houve melhora significativa da membrana neovascular um mês após a injeção foi indicada a realização de fenestração da bainha do nervo óptico à direita para reduzir mais rapidamente o papiledema. Após a cirurgia houve melhora discreta do edema de papila, 4 semanas depois, mas como persistia a membrana neovascular a opção foi pela realização de mais 2 injeções com intervalo de 1 mês entre elas e aumentado ainda mais a dosagem da acetazolamida para $2 \mathrm{~g} / \mathrm{d}$ e de topiramato para $100 \mathrm{mg} 2$ vezes ao dia.

Houve então melhora progressiva do edema de papila, particularmente no OD. Quatro meses após o início do tratamento, 1 mês após a terceira injeção de bevacizumab no OD e 3 meses após a fenestração da bainha do nervo óptico à direita a $\mathrm{AV}$ era de 0,2 no $\mathrm{OD}$ e 1,0 no OE.A paciente persistia com cefaléia discreta e novo campo visual revelou melhora significativa em ambos os olhos, mas persistência de escotomas em ambos os olhos. $\mathrm{O}$ fundo de olho e a angiofluoresceinografia demonstraram melhora significativa do edema de ambos os olhos e da exsudação no pólo posterior do OD (Figura 3). Persistia ainda, no entanto a membrana neovascular sub-retiniana à angiofluoresceinografia (Figura 4) e foi indicado o prosseguimento do tratamento com injeções mensais intravítreas de bevacizumab, na espectativa resolução da membrana neovascular no olho direito.

\section{Discussão}

Pacientes com papiledema frequentemente apresentam sintomas de obscurecimentos transitórios da visão, uni ou bilaterais, com duração de segundos, que estão presentes em até $70 \%$ dos pacientes ${ }^{(7,8)}$. No entanto, de maneira geral a função visual se mostra preservada nos pacientes com papiledema, pelo menos nas suas fases iniciais. Perda definitiva da função visual também pode ocorrer e geralmente se manifesta por defeitos de campo visual que são freqüentes nos pacientes com HII, podendo acometer entre $49-87 \%$ dos pacientes durante 

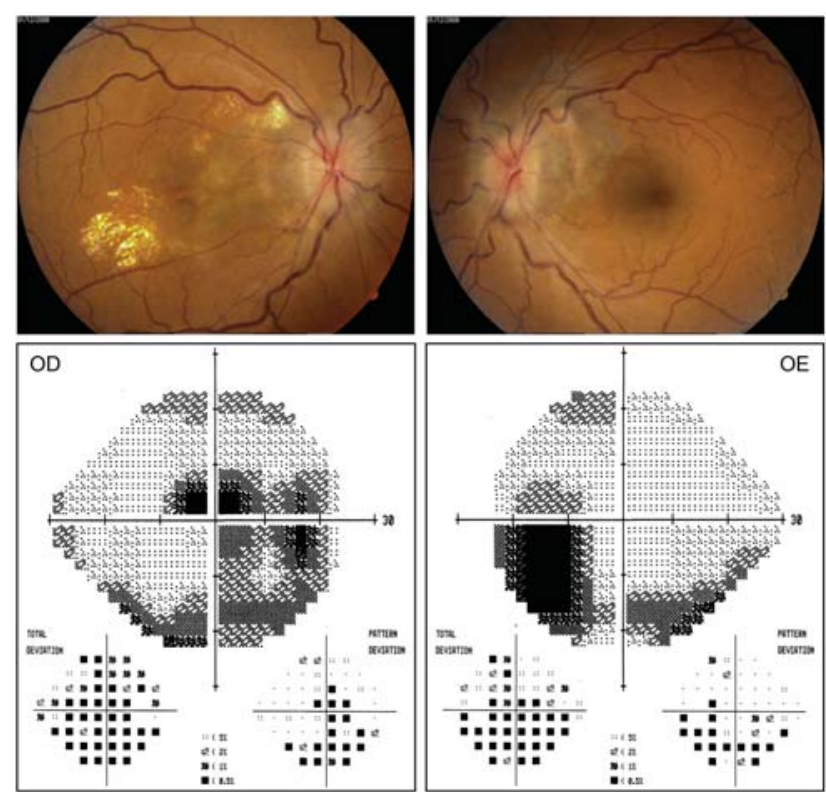

Figura 3: Retinografia demonstrando redução do papiledema em ambos os olhos e da exsudação macular à direita quando comparado ao exame anterior. Abaixo, campo visual computadorizado evidenciando melhora do defeito, mas persistência de escotoma cecocentral no olho direito

alguma fase da doença ${ }^{(2,3,8,9)}$. As alterações campimétricas geralmente são indicativas de perda axonal de evolução crônica sendo mais comuns os defeitos no quadrante nasal inferior, a constrição generalizada do campo visual, os defeitos arqueados e, menos frequentemente, os escotomas centrais ou cecocentrais ${ }^{(10)}$.

O caso atual evidencia uma complicação muito mais rara da HII que é a perda visual decorrente de uma membrana neovascular sub-retiniana. Wendel et al. ${ }^{(11)}$ revisaram 1140 casos de HII e encontraram apenas 6 pacientes com MNSR unilateral, o que sugere uma incidência de apenas $0,53 \%$ dos casos. Não se sabe o mecanismo pelo qual alguns pacientes com papiledema desenvolvem MNDR, mas a existência de vários casos acometendo ambos os olhos ${ }^{(4,6,12)}$ sugere uma predisposição individual para tal ocorrência. A patogênese presumida para tal complicação é que a deformidade provocada pela pressão exercida nas margens da membrana de Bruch ao nível do disco óptico poderia levar a uma descontinuidade da aposição normal das camadas coriorretinianas naquela região que, associada à hipóxia criada pelo edema axonal poderia promover a angiogênese levando à formação da membrana neovascular $^{(6)}$

Devido à raridade da complicação, não se conhece bem a história natural da doença, mas o conceito mais difundido é que a maior parte das MNSR nos pacientes com papiledema são relativamente benignas e perma- necem na região peripapilar ${ }^{(4,11)}$ No entanto, se houver envolvimento foveal e comprometimento do feixe papilomacular pode haver perda visual grave como demonstrado no OD de nossa paciente. Embora no caso atual a perda axonal decorrente do papiledema crônico possa ter contribuído em parte para o desenvolvimento do defeito campimétrico, a redução da acuidade visual e o defeito de campo se deveu fundamentalmente à presença de uma membrana neovascular sub-retiniana que ocasionou redução da acuidade pela distorção dos fotorreceptores secundária a membrana e à exsudação macular no OD, de forma semelhante aquela observada por outros autores na literatura ${ }^{(4-6,11)}$. Com relação ao OE, o defeito de campo pode ter sido decorrente em parte da perda axonal que ocorre no papiledema crônico, mas a análise cuidadosa dos achados à angiofluoresceinografia e indocianina verde indicam que provavelmente tenha existido uma membrana neovascular sub-retiniana peripapilar que involuiu, mas que provocou cicatriz justapapilar seja pela sua presença seja por ter tido hemorragia peripapilar prévia (Figuras 1 e 2).

O tratamento da membrana neovascular no papiledema ainda não é bem estabelecido. Muitos autores já trataram estas membranas com laser de argônio, terapia fotodinâmica ou cirurgia para prevenir a extensão subfoveal ${ }^{(5,6,11,13,14)}$. Jamison ${ }^{(5)}$ relatou uma paciente que desenvolveu membrana neovascular sub-retiniana associada a papiledema em paciente com pseudotumor cerebral. Apesar do tratamento da hipertensão intracraniana ter levado a redução do edema de papila houve progressão da MNSR necessitando tratamento com laser de argônio e levando a redução da acuidade visual. Morse et al. ${ }^{(6)}$ relataram uma paciente com pseudotumor cerebral que desenvolveu MNDR bilateral associada a papiledema. A paciente foi submetida a tratamento do pseudotumor cerebral com melhora do papiledema. Em um olho houve resolução da MNSR, enquanto que no outro houve piora progressiva da membrana, levando a redução da acuidade visual e necessidade de tratamento com laser de argônio. Castellarin et al. ${ }^{(13)}$ realizaram a remoção cirúrgica de uma MNSR em paciente com pseudotumor cerebral, mas não houve melhora na função visual. Mais recentemente, Tewari et al. ${ }^{(14)}$ realizaram tratamento com terapia fotodinâmica combinada com a injeção justaescleral de triamcinolona que resultou em involução da membrana e melhora da acuidade visual. No entanto, esta membrana não se situava na região foveal e se mostrava restrita à região do disco óptico.

Outros autores sugerem que estas membranas podem não necessitar tratamento e que tem um bom prog- 

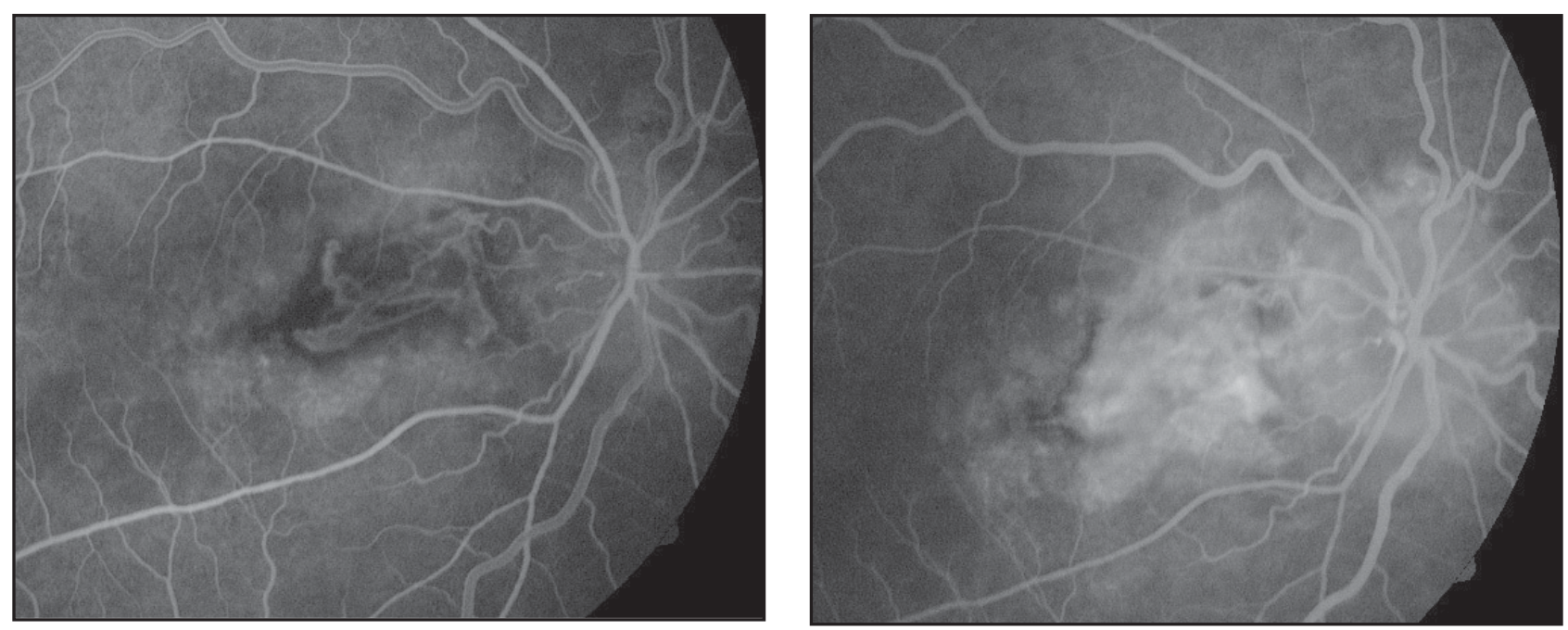

Figura 4: Angiofluoresceinografia do olho direito em fase inicial (à esquerda) e tardia (à direita) evidenciando persistência de membrana neovascular sub-retiniana

nóstico regredindo quando o papiledema melhora ${ }^{(4,11,12,15}$ ${ }^{16)}$. Caballero-Presencia et al. ${ }^{(12)}$ relataram uma paciente com MNSR bilateral que involui após a redução da pressão intracraniana e resolução do papiledema. Coppeto e Monteiro ${ }^{(4)}$ relataram uma paciente com papiledema e MNSR em ambos os olhos que regrediu espontaneamente apesar do pseudotumor ter se mantido recalcitrante. Wendel et al. ${ }^{(1)}$ revisaram 6 pacientes com MNSR e papiledema. Todos realizaram tratamento para o pseudotumor, em 3 foram feitos tratamentos da MNSR com laser de argônio e em outros 3 foram feitos apenas observação da membrana. Os autores concluíram que as MNSR que permanecem restritas à região peripapilar podem ser apenas seguidas, enquanto que aquelas que progridem para a região foveal devem ser tratadas. Avaliando-se sete pacientes previamente publicados que permaneceram sem tratamento específico para a MNSR, 5 tiveram uma boa evolução visual ${ }^{(4,11,12,16)}, 1$ teve extensão da membrana em direção à fóvea ${ }^{(11)} \mathrm{e} 1$ outro que tinha membrana subfoveal teve piora da visão ${ }^{(15)}$.

Na paciente atual a presença de grande membrana já acometendo a região subfoveal e o feixe papilomacular e ocasionando grande baixa da acuidade visual nos levou a procurar um tratamento específico para a MNSR. Como a membrana acometia a região foveal e o feixe papilomacular, procuramos evitar o uso tratamentos que pudessem provocar cicatrização naquelas regiões. Portanto, em paralelo ao tratamento clínico da HII, realizamos injeção intravítrea de bavacizumab acreditando que este poderia levar a melhora da membrana. A opção por este tipo de tratamento foi baseada em estudos prévios com outros tipos de membrana neovascular e no relato de uma paciente com pseudotumor cerebral e MNSR que apresentou resolução completa da membrana após uma única injeção de bevacizumab intravítrea. ${ }^{(17)}$ Como não observamos resultado após a primeira injeção optamos por realizar a fenestração da bainha do nervo óptico no sentido de obter uma resolução mais rápida do papiledema, na expectativa de que a melhora do edema pudesse influir favoravelmente na resolução da membrana. Ainda assim, não houve resolução e procedemos a mais 2 injeções intravítreas da droga antiangiogênica com melhora apenas parcial da membrana e da função visual. É difícil saber a explicação pela qual não obtivemos uma resposta favorável com a injeção de bevacizumab como esperado. No entanto, a análise cuidadosa evidencia discreta melhora da exsudação e do defeito campimétrico (Figura 3) e esperamos que prosseguindo com tal medicação ainda possamos obter a involução da neovascularização.

\section{Conclusão}

O caso atual serve para chamar a atenção para a ocorrência rara de MNSR em pacientes com papiledema e enfatizar que nem sempre a evolução da membrana é favorável mesmo quando se obtém redução do edema do disco óptico. O oftalmologista deve estar atento a esta possibilidade e tratar agressivamente a HII no sentido de resolver o mais rapidamente o papiledema. As membranas pequenas e próximas ao disco podem ser observadas cuidadosamente, mas se apresentarem tendência a crescimento deverão ser tratadas especificamente com 
medicações antiangiogênicas associadas ou não à fotocoagulação com laser de argônio ou com terapia fotodinâmica.

\section{Abstract}

We report a 41-years-old woman with idiopathic intracranial hyperthension (psedotumor cerebri syndrome) that presented with visual loss in one eye due to subretinal neovascular membrane involving the macular area. Despite the introduction of adequate medical treatment followed by optic nerve sheath fenestration that lead to optic disc edema regression there was no improvement in the subretinal neovascular membrane even after performing three sequential intravitreal injection of bevacizumab. We stress the importance of recognizing this uncommon complication of papilledema and review the literature regarding such an occurrence. We also discuss the possible causes for developing subretinal membrane in papilledema, its most common outcome and the treatment modalities available for managing subretinal neovascular membrane in patients with pseudotumor cerebri syndrome. .

Keywords: Pseudotumor cerebri/complications; Retinal neovascularization/physiopathology; Retinal neovascularization/therapy; Papilledema/complications; Visual acuity; Case reports

\section{REFERÊNCIAS}

1. Friedman DI, Jacobson DM. Diagnostic criteria for idiopathic intracranial hypertension. Neurology. 2002;59(10):1492-5.

2. Monteiro MLR. Perda visual na síndrome do pseudotumor cerebral. Arq Bras Oftalmol. 1994;57(2):122-5.

3. Wall M, George D. Visual loss in pseudotumor cerebri. Incidence and defects related to visual field strategy. Arch Neurol. 1987;44(2):170-5

4. Coppeto JR, Monteiro ML. Juxtapapillary subretinal hemorrhages in pseudotumor cerebri. J Clin Neuroophthalmol. 1985;5(1):45-53.

5. Jamison RR. Subretinal neovascularization and papilledema associated with pseudotumor cerebri. Am J Ophthalmol. 1978;85(1):78-81.
6. Morse PH, Leveille AS, Antel JP, Burch JV. Bilateral juxtapapillary subretinal neovascularization associated with pseudotumor cerebri. Am J Ophthalmol. 1981;91(3):312-7.

7. Miller NR. Papilledema. In: Miller NR, Newman NJ, editors. Walsh \& Hoyt's Clinical Neuro-Ophthalmology. 5th ed. Baltimore: Williams \& Wilkins Baltimore; 1998. p. 487-548.

8. Wall M, George D. Idiopathic intracranial hypertension. A prospective study of 50 patients. Brain. 1991;114(Pt 1A):155-80.

9. Corbett JJ, Savino PJ, Thompson HS, Kansu T, Schatz NJ, Orr LS, Hopson D. Visual loss in pseudotumor cerebri. Follow-up of 57 patients from five to 41 years and a profile of 14 patients with permanent severe visual loss. Arch Neurol. 1982;39(8):461-74.

10. Monteiro MLR, Moura FC. Aspectos oftalmológicos da síndrome da hipertensão intracraniana idiopática (pseudotumor cerebral): [revisão]. Rev Bras Oftalmol. 2008;67(4):196-203.

11. Wendel L, Lee AG, Boldt HC, Kardon RH, Wall M. Subretinal neovascular membrane in idiopathic intracranial hypertension. Am J Ophthalmol. 2006;141(3):573-4.

12. Caballero-Presencia A, Diaz-Guia E, Martinez-Perez M, Lopez-Lopez JM. [Bilateral juxtapapillary subretinal neovascularization in a case of pseudotumor cerebri]. J Fr Ophtalmol. 1986;9(2):105-10. French.

13. Castellarin AA, Sugino IK, Nasir M, Zarbin MA. Clinicopathological correlation of an excised choroidal neovascular membrane in pseudotumour cerebri. Br J Ophthalmol. 1997;81(11):994-1000.

14. Tewari A, Shah GK, Dhalla MS, Shepherd JB. Combination photodynamic therapy and juxtascleral triamcinolone acetonide for the treatment of a peripapillary choroidal neovascular membrane associated with papilloedema. $\mathrm{Br} \mathrm{J}$ Ophthalmol. 2006;90(10):1323-4.

15. Akova YA, Kansu T, Yazar Z, Atabay C, Karagöz Y, Duman S. Macular subretinal neovascular membrane associated with pseudotumor cerebri. J Neuroophthalmol. 1994;14(4):193-5.

16. Troost BT, Sufit RL, Grand MG. Sudden monocular visual loss in pseudotumor cerebri. Arch Neurol. 1979;36(7):440-2.

17. Jamerson SC, Arunagiri G, Ellis BD, Leys MJ. Intravitreal bevacizumab for the treatment of choroidal neovascularization secondary to pseudotumor cerebri. Int Ophthalmol. 2008 Jan 15. [Epub ahead of print].

\section{ENDEREÇO PARA CORRESPONDÊNCIA: \\ Mário Luiz Ribeiro Monteiro \\ Av. Angélica 1757 - Conj. 61 \\ CEP 01227-200 - São Paulo - SP - Brasil \\ e-mail: mlrmonteiro@terra.com.br}

